\title{
Cultura de reciprocidad en economías campesinas: una breve exploración de su vigencia de dos comunidades del departamento de Caaguazú
}

\section{Culture of reciprocity in peasant economies: a brief exploration of its validity of two communities in the department of Caaguazú}

\author{
Quintín Riquelme ${ }^{a}$, Elsy Vera ${ }^{a}$
}

\begin{abstract}
Resumen
El trabajo sobre cultura de reciprocidad explora en dos comunidades del departamento de Caaguazú cómo funciona la economía campesina. La misma está inserta dentro del mercado pero internamente opera una red de relaciones -reciprocidad- en la cual la racionalidad que supone el mercado tiene una presencia relativa. La reciprocidad en Paraguay fue estudiada principalmente por autores como: Meliá, Fógel, Carbonell de Masy, Lehner et al., entre otros. El objetivo es describir y analizar la reciprocidad como una cultura arraigada en las economías campesinas cuyos principios son la cooperación, lo ético y lo colectivo, frente a la competencia, lo material y lo individual de la economía mercantil. La metodología es de tipo exploratorio con enfoque cualitativo y cuantitativo y corte trasversal. La reciprocidad expresada en el jopói, la minga y el trueque, sigue siendo una práctica habitual en las comunidades campesinas, pese a la creciente mercantilización de su economía.
\end{abstract}

Palabras clave: reciprocidad, economía campesina, el don, jopói, minga, trueque, relaciones de cooperación, mercantilización.

\begin{abstract}
This paper explores how a culture of reciprocity functions in the rural economy of two communities in the Department of Caaguazú, Paraguay. While the community is inserted in the market, an internal network of relationships -reciprocity- operates in a way that the presumed rationale of the market has a minor presence. Reciprocity in Paraguay has been studied primarily by authors such as: Meliá, Fógel, Carbonell de Masy, Lehner et al., among others. The objective is to describe and analyze reciprocity as a culture that is rooted in rural economies whose principles are cooperative, ethical, and collective, rather than competitive, material, and individualistic
\end{abstract}

a Universidad Nacional de Asunción, Facultad de Ciencias Sociales, Paraguay.

Correspondencia a: quintinriquelme@gmail.com

Recibido:

3 setiembre 2018

Aceptado:

26 octubre 2018

Artículo publicado en acceso abierto bajo la Licencia Creative Commons.

\section{(c) (7)}

Cita:

Riquelme, Q. \& Vera, E. (2018). Cultura de reciprocidad en economías campesinas: una breve exploración de su vigencia de dos comunidades del departamento de Caaguazú. Kera Yvoty: reflexiones sobre la cuestión social, 3, 9-20. 
Riquelme, Q. \& Vera, E. Cultura de reciprocidad en economías campesinas: Una breve exploración de su vigencia de dos comunidades del departamento de Caaguazú.

as in a mercantile economy. An exploratory methodology is used with a qualitative, quantitative and crosscutting focus. Reciprocity, such as jopói (lending a hand), la minga (community work groups) and bartering continue to be regular practices in rural communities, despite the growing mercantilization of their economy.

Keywords: reciprocity, rural economy, giving, jopoi, community work, bartering, cooperative relationships, mercantilization.

\section{Introducción}

La presente investigación aborda la reciprocidad en comunidades campesinas y busca fundamentalmente relevar si la economía de reciprocidad tal como la definió la antropología para las sociedades tribales como la reproducción del don (Temple, 1989), es un concepto y una práctica vigente en las comunidades campesinas. Si esas relaciones de cooperación tan frecuentes como los intercambios de implementos, de comida, de ayuda a algún miembro de la comunidad en momentos difíciles, pueden ser asimiladas dentro del concepto de reciprocidad y si dichas prácticas son posibles sostenerlas en comunidades $\mathrm{o}$ sociedades cada vez más complejizadas e invadidas por el intercambio de carácter mercantil.

Las familias campesinas en Paraguay están inmersas en una compleja red de relaciones que resulta difícil desentrañar sin una observación en profundidad por los elementos que la componen: económicos, sociales y culturales. Los antropólogos han develado unas formas de relacionamiento y unas prácticas económicas en las sociedades tribales distintas a las de las sociedades occidentales. Es la llamada economía de reciprocidad en la cual el principio fundamental no es el interés privado, sino el bien común. Es la reproducción del don y es antagónica a la economía del intercambio mercantil.

Lacontribución delaantropología para el conocimiento de procesos económicos, sociales, culturales y políticos de los pueblos primitivos es de mucho valor también para la comprensión de estos mismos procesos en las comunidades campesinas actuales. Tal vez la gran diferencia entre ambas sociedades está en el sistema de tenencia de la tierra como lo señalara Georg Grunberg (1975). El sistema prevaleciente en las sociedades indígenas es el colectivo, mientras que en las comunidades campesinas es el individual en el régimen de la propiedad privada, aunque los campos comunales en las colonias antiguas y las parcelas comunitarias en los asentamientos nuevos constituyen espacios de socialización de los y las pobladores de la comunidad.

Una aproximación al conocimiento de la economía de reciprocidad parte de la afirmación de que la misma es antagónica a la economía basada en el intercambio mercantil. "Este antagonismo se manifiesta en el hecho de que el intercambio se justifica a partir de la necesidad individual y la reciprocidad a partir de la necesidad del otro" (Temple, 1989, p. 21).

El mismo autor afirma que en 1922 en Los Argonautas del Pacífico, Bronislaw Malinowski, redescubrió la economía del don y Marcel Mauss, al año siguiente generaliza sus observaciones en las sociedades llamadas por él arcaicas. Con esas observaciones dice Temple (2003), se destruye la fábula del homo economicus, en la cual se sostenía que la principal inquietud de los individuos es procurarse los bienes que les son necesarios. En los inicios de la era industrial "los teóricos de la economía política habían imaginado que toda economía reposa sobre el intercambio mercantil" (p. 1). Sin embargo, el don está en el principio de reconocimiento del otro. Se reconoce al otro como parte de una entidad común que se llama humanidad. Se fundamenta en el valor ético (Temple, 1989, p. 21). 
A partir del principio del don y de su reproducción, que llamamos reciprocidad, resulta en seguida que quien da más dentro de una comunidad, participa más del ser humano [...] Se puede decir que hay fundamentalmente una equivalencia entre el don y la adquisición del ser, del prestigio o renombre: hay una relación directa y sistemática entre el don y el renombre lo que inmediatamente se traduce en poder. El prestigio viene a ser entonces el nombre mismo del poder [...] Esta equivalencia entre el don y el prestigio explica que quien reivindica la palabra y el título de jefe tiene que dar más que los otros para merecerla". (Temple, 1989, p. 22)

Adam Smith, observaba (Temple, 2003) cómo sus contemporáneos calculaban todo por interés y que existe una tendencia natural del hombre a traficar e intercambiar sólo en su propio interés. Reproduciendo una frase de Smith expresa:

Por ejemplo, en una tribu de cazadores o pastores, un individuo hace arcos y flechas con más celeridad y destreza que otro. Trocará frecuentemente esos objetos con sus compañeros por ganado o caza y no tardará en darse cuenta de que, por este medio, podrá procurarse más ganado y caza que si él mismo fuera a cazar. Por cálculo de interés, entonces, convierte la fabricación de arcos y flechas en su principal ocupación. (p. 1)

Si Adam Smith hubiera observado una sociedad de cazadores de verdad, dice Temple (2003), "antes que construir un relato-ficción, habría constatado que el arco o la caza, incluso producidos en demasía por el trabajo del cazador, nunca es intercambiado sino siempre donado". Agrega que la "génesis del ser social es inmediatamente la razón de una economía humana, ya que si hay que donar para ser, para donar hay que producir". De éstas observaciones concluye, que la "reciprocidad de dones no es, pues, una forma arcaica del intercambio; ella es otro principio de la economía y de la vida" (p. 1).

Esta reflexión, dice Temple (1987) permitiría fundar la sociedad como un sistema económico-político en la cual la "reproducción del don connota socialidad y asimismo traduce su comprensión social y define el contorno genérico de la humanidad".

Meliá (1994) destaca que dentro de la economía de reciprocidad hay dos tipos de relaciones:

-los movimientos de viceversas que también son llamadas simétricas y;

-los movimientos centralizados

La primera se da entre dos elementos. Está marcada por la dualidad y la simetría. "Consiste en el hecho de que uno da al otro, lo que éste necesita, ni más ni menos; es decir, sin dar más para aplastarlo y someterlo" (Temple, 1989).

La segunda, cuando participa un grupo. Se le denomina también economía de la comunidad o comunidad de redistribución. En una comunidad de redistribución, los alimentos son los que más comúnmente se distribuyen; esta distribución está asociada al cacicazgo; y las formas de dar son diversas y generalmente van acompañadas de prácticas rituales.

La reciprocidad igualmente tiene otros elementos:

-La interacción, que es la reciprocidad propiamente dicha;

-Las relaciones sociales que se dan entre el donante y el donador; $y$

-Lo material, la materia, las circunstancias materiales. Lo que se da es muy importante y define el tipo de relación. (Meliá, 1994, p. 36)

Estos tres elementos configuran la 
Riquelme, Q. \& Vera, E. Cultura de reciprocidad en economías campesinas: Una breve exploración de su vigencia de dos comunidades del departamento de Caaguazú.

Economía de reciprocidad y se dan en forma continua. En un extremo se encuentra el don puro, la dación pura, llamada también la reciprocidad positiva definida "como una reciprocidad en la cual el don es la medida del poder" (Temple, 1989, p. 125). En el otro extremo podría darse hasta la venganza pura, es decir la reciprocidad negativa.

El don puro, es la ayuda dada libremente sin exigir devolución o retribución. La reciprocidad negativa en cambio es la apropiación indebida, egoísta (Meliá, 1994).

La tierra es el soporte fundamental para la economía de reciprocidad que se resuelve paradigmáticamente en la fiesta, la forma de vida a la que el guaraní aspira como plenitud (Meliá \& Temple, 2004, p. 24).

Para la economía campesina, la tierra también es el principal soporte. Es el medio de producción y de reproducción de la vida. El acceso a ella asegura la satisfacción de las necesidades básicas como la vivienda, el trabajo, la alimentación, la educación, además de las formas culturales y su modo de vida. Es fundamento de su propia vida y la de su familia. Les asegura la subsistencia, es su trabajo, es su mayor capital. En general, el campesino y la campesina no se aferra a la tierra sólo por una cuestión de propiedad, sino porque siente que en la tierra se encuentran sus raíces, que en ella produce, que allí está su historia, su pasado, pero también su futuro y el de los hijos e hijas (Van Dam, 2007).

Galeano (1984) al estudiar las actitudes de los campesinos ante la tierra descubre que éstas, están basadas, por un lado, en:

[...] elementos culturales extraeconómicos, como la posibilidad de vivir en familia, rodearse de amigos, de asumir la responsabilidad de la producción y de ser el señor de sí mismo. Y por otro lado, en elementos basados en la lógica de la economía campesina, en la que prevalece la explotación propia, donde desplegar sus conocimientos y destrezas. (p. 35)
A partir de estos dos elementos contrapuestos concluye que:

[...] la cultura campesina a lo largo de su formación histórica es la resultante de la combinación de elementos inherentes y de elementos derivados o externos[...] yestoporquela población campesina no es homogénea y se enfrenta a los distintos procesos de cambios desde diferentes posiciones y de distinta manera. (Galeano, 1984, p. 35)

También concluye que, "debido al predominio cada vez mayor de la lógica de la economía de mercado (capitalista) los campesinos comienzan a percibir y a utilizar la tierra como un bien comercial" (Galeano, 1984, p. 35).

Esta combinación de intereses y de visiones de los/as campesinos/ as sobre la tierra es producto de la mayor mercantilización impuesta por el capitalismo en el campo, que gradualmente va generando contradicciones en el campesinado. Al mismo tiempo, la mayor valorización de la tierra va haciendo más complicado el acceso a la misma. Hoy, sin la participación del Estado, el acceso del campesino a la tierra se vuelve imposible.

Para lacomprensióndela reciprocidad en las comunidades campesinas es importante caracterizar su sistema de vida.

Una primera y fundamental es su vínculo con la tierra, el campesino y la campesina vive en su tierra y vive de su tierra, por lo tanto, el cuidado del ambiente y la conservación de la biodiversidad son fundamentales para su reproducción. Esta principal característica lo diferencia del empresario agrícola que sólo vive de su tierra, buscando sacar el mayor provecho económico de ella, por lo tanto el interés por conservar la biodiversidad y mantener saludable el ambiente no es su interés primordial.

Otra es la diversidad productiva, produce los principales rubros de su alimentación diaria, además de animales 
menores. En esta lógica de producción, la utilización de productos químicos -fertilizantes y plaguicidas- no puede ser parte de su sistema productivo porque si los emplea estaría destruyéndolo. Por esta razón, se puede afirmar que la producción diversificada es el principal enemigo de los nuevos paquetes tecnológicos que quiere imponer la agricultura empresarial, que con ello, busca además homogeneizar el consumo.

También las unidades de producción campesinas y las comunidades rurales en general, producen y reproducen múltiples relaciones: de cooperación, de reciprocidad, de solidaridad, como la minga, el jopói, el trueque y otro tipo de relaciones generado por el parentesco, el compadrazgo, la vecindad, etc, independientemente de las características socioeconómicas de dichos actores. Una relación de tipo horizontal (Riquelme, 2014).

Pero es importante destacar por otro lado, que las unidades campesinas no son homogéneas.

Dependiendo del territorio que ocupa, pueden ser caracterizadas de una u otra manera. Apelando a la clasificación planteada por Ramiro Domínguez, en su libro El Valle y la Loma, el campesino perteneciente al poblado Valle, es más tradicional, está inmerso en una economía agrícola-ganadera. En cambio el campesino perteneciente al poblado Loma, es más dinámico, practica una economía centrada al mercado (Domínguez, 1977).

Deigual manera, Eric Wolf (1977) hace una diferenciación entre los campesinos que viven en comunidades corporativas $\mathrm{y}$ campesinos que viven en comunidades abiertas. Las comunidades corporativas están aferradas a mantener cierto equilibrio tradicional, tratando de minimizar o evitar las influencias externas que amenazan la integridad de su comunidad. En cambio las comunidades abiertas promocionan la interacción con el mundo exterior, "permite la acumulación individual y el consumo de riquezas durante los períodos de incremento de la demanda externa”.
Dependiendo de la extensión de las parcelas ocupadas y de la capacidad productiva, se encontrarán diversos estratos de campesinos, a partir de los cuales se podrá construir una tipología: los muy pobres, los pobres y los que tienen un nivel aceptable, los llamados medianos productores.

De esta manera ser campesino, es mucho más que "pequeño productor que hace relación a una escala y agricultura familiar a una economía, el campesino designa un ethos y una clase, de modo que reconocerse campesino es el primer paso en el camino de reafirmar una específica socialidad y -eventualamente- conformar un sujeto colectivo de primera división" (Bartra Verges, 2010).

En términos sociales, el campesino es un conglomerado social en cuya base está la economía familiar multiactiva pero del que forman parte también y por derecho propio, quienes teniendo funciones no directamente agrícolas participan de la forma de vida comunitaria y comparten el destino de los labradores. El campesino es un modo de vida, entender esto es esencial para comprender su naturaleza (Bartra Verges, 2010).

El objetivo del trabajo es describir y analizar las relaciones de cooperación como una cultura arraigada en las economías campesinas y como una forma de resistencia frente a la economía de mercado que privilegia la competencia, lo material y lo individual, frente a la cooperación, lo ético y lo colectivo. En lo concreto se buscará: $a$. Relevar y describir las prácticas de reciprocidad como el jopói, la minga y el trueque; $b$. Analizar si la reciprocidad forma parte del imaginario campesino y en qué medida contribuye a la construcción o al fortalecimiento de su identidad; $y, \quad$ c. Realizar un análisis comparativo de las prácticas de reciprocidad en una comunidad antigua y otra más reciente.

\section{Materiales y Métodos}

La metodología aplicada es de tipo exploratorio con enfoque cualitativo y 
Riquelme, Q. \& Vera, E. Cultura de reciprocidad en economías campesinas: Una breve exploración de su vigencia de dos comunidades del departamento de Caaguazú.

también cuantitativo de corte trasversal. En lo cuantitativo se buscó demostrar que en la cotidianeidad de las familias campesinas se dan diversas situaciones, intercambio de productos y alimentos entre parientes y vecinos, intercambio de trabajo uno o dos días a la semana, préstamos mutuos de implementos agrícolas, etc., de las cuales no se tienen datos, estadísticas. De existir esas prácticas existen, pero con qué grado de intensidad, cantidad y frecuencia. Estas son estrategias o prácticas arraigadas en las comunidades campesinas, trasmitidas de generación en generación, pero, hace falta analizarlas con mayor detalle, qué intercambian, cómo intercambian y con quién intercambian. Si las relaciones de reciprocidad o de cooperación en las familias campesinas se analizan desde el ámbito de la cultura, no hay duda que es una práctica que tiene su origen en los antepasados, pero si se analiza desde el punto de vista socioeconómico, dichas prácticas formarían parte de las múltiples estrategias de supervivencia para afrontar las dificultades de pobreza, de exclusión y de explotación que sufren las familias campesinas.

En lo cualitativo, se analizó si estas prácticas forman parte del imaginario campesino, en qué medida es un factor que mantiene y reproduce su identidad y también en qué medida puede ser catalogada como una forma de resistencia contra la hegemonía de los mercados, que pretende imponer el capitalismo y cuál es la percepción con relación a esas prácticas.

La exploración fue realizada en dos comunidades del departamento de Caaguazú, una antigua conocida como Jakarei en el municipio de Caaguazú, fundada en la década de 1960 en el marco de la política de reforma agraria y otra reciente, un asentamiento creado en el 2008 llamado María Cristina en el municipio de Yhú. En cada comunidad se realizaron 10 entrevistas a igual número de familias, para las cuales se contó con una guía semi-estructurada y una encuesta básica que permitió recoger información cualitativa y cuantitativa de las familias seleccionadas para dicha finalidad. Las preguntas que guiaron la investigación fueron:

1. ¿La reciprocidad tal como la define la antropología es una práctica presente en las comunidades campesinas?

2. ¿La reciprocidad se da de igual manera en las comunidades campesinas tradicionales como en los nuevos asentamientos?

3. ¿Es posible sostener esta práctica en una economía cada vez más mercantilizada, en la cual las posibilidades de compartir se vuelven cada vez más difíciles?

La reciprocidad es definida en este estudio como toda relación de cooperación no mediada por monedas entre familiares y vecinos en una localidad determinada. Es una práctica propia de comunidades primitivas transmitidas de generación en genración. Las comunidades campesinas no están ajenas a esta práctica. Son básicamente el jopói, la minga y el trueque.

\section{Resultados \\ La reciprocidad en las comunidades seleccionadas}

La reciprocidad como concepto analizado por los antropólogos para explicar el funcionamiento de las economías indígenas, quizás no tenga la misma connotación para explicar estos fenómenos en las economías campesinas, sin embargo, es posible afirmar que muchas de las prácticas relevadas de las comunidades indígenas se reproducen en las comunidades campesinas, no con la misma intensidad, ni con la misma cuantía, pero el espíritu solidario, la cooperación sin mediación de la moneda se da de manera habitual.

Las relaciones de cooperación entre familias vecinas y parientes como es sabido tienen raíces históricas. Un estudio realizado por la Pastoral Social de la Conferencia Episcopal Paraguaya (1984) sostenía, que la eficacia relativa de las economías campesinas 
se reforzaba con la vigencia de relaciones solidarias entre ellas, herencia histórica de la organización social de los guaraníes. Estas relaciones solidarias se sustentan en el "intercambio solidario de servicios que permiten una estrecha articulación de las pequeñas unidades minifundiarias alrededor de las unidades productivas de mediana envergadura" ( $p, 360)$.

En los asentamientos campesinos, sobre todo en aquellos que fueron resultado de largos procesos de disputa, el Estado generalmente entrega la tierra y deja abandonado/a a los /as beneficiarios/as. En estos casos la reciprocidad, la cooperación es la única vía de sobrevivencia, no existe otra, hasta que comienza la cosecha de los primeros rubros sembrados, especialmente el maíz, el maní y la mandioca.

En el asentamiento María Cristina, en los primeros meses las familias vivían de la cooperación interna y externa, recién después de dos años el Estado comenzó a brindar asistencia más sistemática, en caminos, escuelas, energía eléctrica, entre otras.

Cuando vinimos, los que estábamos un poco mejor, si venía una persona a quien se le dio un lote, por lo menos un año le damos lo que tenemos como mandioca, poroto, maíz. Nos ayudamos en ese sentido por un año y en el siguiente año esa persona ya tiene que preparar la producción para su consumo, ese el compromiso. En esos aspectos por lo menos nos salvamos y nos ayudamos, acá si hay enfermos, si hay mucha necesidad, nosotros nos reunimos aunque sea en la iglesia, en la escuela o hacemos reunión cada 15 días que es general, ahí vemos propuestas para ver cómo vamos a ayudar a esa persona. En primer lugar vemos qué posibilidad tiene de mantenerse por sí sola, si no tiene posibilidad nos responsabilizamos por ella en este caso. $\left(\mathrm{E} \mathrm{N}^{\circ} 1\right)$

\section{El Jopói}

El jopói en su expresión literal es tender la mano al que necesita. Simboliza solidaridad, ayuda mutua. Es la que más se practica en las comunidades. Si bien los y las entrevistados/as reconocen una fuerte influencia de la cultura moderna, el jopói sigue ocupando un espacio importante en las relaciones comunitarias.

Tanto en el asentamiento María Cristina, como en la colonia Jakare'i, todas las familias entrevistadas, 20 en total, reconocen el jopói como una práctica habitual que en algunos casos se da incluso diariamente. Las cosas donadas con mayor frecuencia son las comidas, los productos de la chacra y de la huerta.

En los dos lugares, el sujeto del jopói son los parientes y los vecinos y entre éstos generalmente el más pobre y sobre todo cuando algún miembro de la familia está afectado por alguna discapacidad. En estos casos el jopói es casi diario.

En cuanto a frecuencia, sobresalen los intercambios semanales, en solo dos casos el jopói es diario, en uno por la presencia de una discapacitada en uno de los hogares y en otro por la cercanía con los hijos. El jopói es una práctica que tiene a la mujer como protagonista. Son las que conocen las necesidades de sus vecinas y porque también conocen cuando faltan o escasean los artículos de primera necesidad utilizados en la preparación de las comidas. Los hombres participan escasamente debido al rol que desempeña en la familia, éstos trabajan en la chacra o fuera de la comunidad en algunos casos, por lo tanto tienen menor o escasa injerencia en las tareas del hogar.

Es un tema muy dificil hoy en día porque hay gente solidaria, jopói es solidaridad y el jopoi -o jepoi- hace la gente sin verguenza y esto le afecta a la gente solidaria. El jopói es un valor muy importante, en nuestra base San Isidro (Jakarei) todavía se da porque esta comunidad es muy religiosa. Acá a los enfermos se les visita se les hace 
Riquelme, Q. \& Vera, E. Cultura de reciprocidad en economías campesinas: Una breve exploración de su vigencia de dos comunidades del departamento de Caaguazú.

colecta, se les da alimentos, queso, huevos, entre otras cosas. Y también hay personas que no se esconden de la gente a la hora de la comida. (E $\mathrm{N}^{\circ}$ $11 \mathrm{CJ})$

\section{La minga}

La minga es otra forma de cooperación, es conocida comúnmente como intercambio de trabajo. La minga es una práctica que va desapareciendo por la mercantilización cada vez más creciente de la economía. Pero en la mayoría de las comunidades se siguen practicando aunque no con la intensidad de décadas anteriores. De las 10 entrevistas en María Cristina, cuatro familias respondieron que practican y en un caso la frecuencia es diaria entre dos vecinos en la preparación de carbón y para todas las otras labores agrícolas. Un caso particular es el cuidado mutuo de los hijos entre dos familias, cuando por alguna actividad laboral fuera del hogar deben dejar sus hijos.

Y hacemos algunas veces pero no continuado, no es solo eso, más bien le ayudamos al que más necesita. Hacemos la minga pero un 20 a $30 \%$ nomas y después más bien le ayudamos si vemos que no va a poder hacer solo. Eso hacemos constantemente, este tipo de trabajo para ser un ejemplo para los demás. $\left(\mathrm{E} \mathrm{N}^{\circ} 1, \mathrm{AMC}\right)$

En la colonia Jakare'i siete familias respondieron positivamente a la práctica de la minga, especialmente en la etapa de preparación de suelo para la siembra. El intercambio se da entre arada por corpida o carpida. En comunidades con escasez de bueyes y arados, el intercambio durante la preparación de suelo por alguna otra actividad es habitual. Es importante destacarqueentiemposdeescasezescuando mayor debería haber sido esta práctica, sin embargo, esa costumbre de ayudarse mutuamente en las labores agrícolas va desapareciendo paulatinamente por la mayor mercantilización de las economías.

\section{El trueque}

El trueque conocido también como permuta, es intercambio de bienes $o$ servicios sin la intervención del dinero, cambio directo. El trueque es una modalidad de cooperación en la cual el intercambio es entre equivalentes. Las cosas intercambiadas deben tener valores similares, medidas generalmente en precios y en peso. En el asentamiento María Cristina sólo cuatro entrevistados/as respondieron afirmativamente. El trueque era una modalidad de cooperación común en las comunidades campesinas y suple la falta o escasez de circulantes. Su frecuencia es de algunas veces al año, aunque había en un caso concreto de frecuencia semanal, el trueque de leña por algunos alimentos.

\section{Discusión \\ La reciprocidad en Paraguay}

Meliá y Temple (2004, p. 237) afirman que "la antropología nos enseña en primer lugar que antes de estar sometidas al libre cambio, todas las comunidades se organizaban según las diferentes estructuras de reciprocidad".

En Paraguay, las décadas de 1960 y 1970 marca un punto de inflexión en la economía campesina. La estructura agraria tradicional caracterizada por el minifundio y latifundio y por el predominio de una agricultura parcelario comunitaria (PSN, 1984) comienza a sufrir cambios significativos con el avance de la llamada modernización agraria. De una agricultura mayoritariamente de subsistencia se pasa a una progresiva mercantilización de la misma. Las nuevas propuestas tecnológicas con la incorporación de los insumos químicos dejaron huellas imborrables sobre la economía campesina.

Los efectos en las comunidades no tardaron en producirse. Con el predominio de la agricultura de subsistencia, las relaciones de cooperación, de ayuda 
mutua, eran mucho más intensas por la homogeneización de la estructura agraria y de las necesidades. Los servicios básicos como agua potable, energía, eran inexistentes y la construcción de infraestructuras para escuelas, caminos, capillas, etc., eran asumidas como obligaciones propias de las comunidades. Con un estado mínimo y lejano de las comunidades, la cooperación y la organización eran necesidades ineludibles para hacer funcionar la sociedad local como tal.

Con la modernización agraria de alguna manera se rompe con esa tradición porque incorpora nuevas exigencias, nuevos estilos de vida que confronta con la tradición. En lo productivo las familias son exigidas a someterse a las reglas del mercado financiero y a paquetes tecnológicos a los que no estaban acostumbradas. La presencia del Estado a través de algunas de sus instituciones comienza a tener influencia y promueve cambios en la forma tradicional de la práctica agrícola y un mayor acercamiento a las reglas del mercado.

La introducción de relaciones económicas basadas en el mercado, dice Carbonell de Masi (1980) choca con el mundo originario del campesino, con su sistema tradicional y le produce una suerte de crisis que en la mayoría de los casos resuelve con la migración. No puede seguir con su costumbre y su sistema de producción tradicional y tampoco asimila completamente lo moderno porque eso implica lógica distinta. Antes, la ayuda recíproca permitía un intercambio amistoso de prestaciones mutuas sin mediciones, ahora las prestaciones de servicios se evalúan sobre la base del dinero. Cuando no dispone de dinero ya no recurre a las prestaciones y viceversa.

Además de las actividades con mayor visibilidad como la construcción y reparación de caminos, de escuelas, que precisan de la cooperación comunitaria, así como las diversas formas de recreación, se dan otras relaciones de cooperación y de reciprocidad menos perceptibles desarrolladas en los niveles más internos e íntimos entre familiares y vecinos. Estas son: el jopói, la minga y el trueque.

Todos estos espacios intercambios dice Gianinazzi en el prólogo del libro (Lehner, Pilz \& Riquelme, 2004).

generarredesnodeberíaserrestringida a la brutalidad de lo económico, ni a la violencia de las asimetrías de poder, ambas obviamente presentes en el intercambio[...] El investigadordeberá intentar descentrar, desviar por lo menos temporalmente estas fuerzas [...] para llegar por lo menos fugaz y momentáneamente a sentir estos territorios simbólicos e imaginarios inmensos que fluyen más allá del intercambio posible delimitado por lo económico y lo ideológico, para explorar lo que realmente están intercambiando estas personas, dentro de este campo interpersonal, campo de batalla intensísimo de proyección, introyección, estrategias de defensa, identificación, sumisión, etc. (p. 3 )

En Paraguay en el 2008 existían un poco más de 288 mil fincas de las cuales cerca del 93\% (CAN 2008) corresponde a la agricultura familiar campesina de hasta 50 hectáreas. La agricultura campesina por tanto, tiene un peso cuantitativo y cualitativo importante, por su cantidad, por ser productora y proveedora de alimentos a la población y también por constituirse en depositaria de una cultura basada en la reciprocidad, en el sostenimiento y mejor uso de los recursos naturales (Riquelme \& Vera, 2016). Es común, sin embargo, encontrar programas o proyectos que desconocen la tradición cultural de las familias campesinas y tratan de implantar políticas públicas y sociales desconociendo estas formas de vida y obviando la participación. Desde este punto de vista, la investigación podrá aportar conocimientos para que las políticas públicas dirigidas al 
Riquelme, Q. \& Vera, E. Cultura de reciprocidad en economías campesinas: Una breve exploración de su vigencia de dos comunidades del departamento de Caaguazú.

sector consideren la importancia de estas particularidades y potencialidades.

\section{Conclusiones}

¿Jopói ojopoi. Cultura de reciprocidad o cultura de la indiferencia?

Los antropólogos han observado y analizado la reciprocidad en las comunidades tribales. Muchas de esas prácticas probablemente han desaparecido o se han reducido en las propias comunidades indígenas actuales por los profundos cambios operados en dichas formaciones sociales, pero, se conoce por la trasmisión oral y por las observaciones directas que la reciprocidad, una forma diferente de práctica económica que genera a su vez relaciones sociales diferentes a la que proponen la economía política moderna sigue siendo habitual en tales economías e incluso en las comunidades campesinas.

La vida cotidiana de las familias campesinas está marcada y trascurre aún en el marco de esa costumbre de compartir, muchas de ellas realizadas en un espacio reducido, entre vecinos y parientes cercanos, razón por la cual no se visibilizan y porque además no tiene ese carácter prebendario-clientelista propias de sociedades mercantilizadas. Se fundamenta en el valor ético-religioso, en la cultura de la solidaridad, de projimidad, que va más allá de la materialidad y del efecto de mostración. Los antropólogos concuerdan que el compartir cosas materiales, el dar, van acompañadas de un espíritu que Mauss (1971) llamó el maná que es el mismo ser -el alma- del donador inserta en la cosa donada y que en la cultura campesina podríamos llamarlo projimidad, jehecha cua'a -no desconocer la necesidad del otro-, solidaridad.

Esta cultura de projimidad, de solidaridad, desde un tiempo a esta parte está siendo avasallada por otra cultura que pretende imponer y que de hecho ha impuesto el capitalismo, el intercambio mercantil dentro del cual todo se mide en valores de cambio. Jopói o Jopoi/jepoi - dejar de hacer- es la disputa en el marco de las nuevas relaciones sociales impuestas por el neoliberalismo. Las familias campesinas están sometidas a fuertes presiones de modificación de su modo de vida, desde la pérdida de sus tierras, sus semillas, su hábito de consumo y su manera de relacionarse con su entorno inmediato.

En las entrevistas a las familias, la respuesta o la reacción inmediata ante la pregunta del jopói es, lo que existe ahora es el jopoi-jepoi y no el jopói. Sin embargo, urgando un poco más con las preguntas, reconocen la existencia del jopói, es más, en algunos casos es bastante intenso y se da incluso casos de prácticas diarias cuando se trata de familias muy pobres. Sólo que con los muy pobres la reciprocidad se da en una sola dirección, no existe el retorno porque el/a que recibe no tiene con qué retornar, pero esta unidireccionalidad en el dar no produce el rompimiento de las relaciones, hay conciencia de que aquel o aquella que recibe no está en condiciones de reproducir el gesto en cosas materiales, pero sí en gestos de agradecimiento, de predisposición a la colaboración, entre otros.

Cuando se lanza la pregunta sobre estas modalidades de cooperación, la mayoría de los y las entrevistados/as recupera el antes, cómo era con sus padres y sus abuelos y recuerda con cierta nostalgia esa época pasada en la cual, la cooperación familiar y comunitaria era muy extendida. La cooperación llega a su máxima expresión en los festejos patronales. La donación de cosas es abundante para la fiesta y del festejo participan todos los miembros de la comunidad, incluso de las comunidades vecinas.

Un ejemplo compartido por un entrevistado narra que en la fiesta patronal las familias donan gallinas, cerdos, novillos, huevos, quesos y todo lo que haga falta. Esta costumbre es aún muy arraigada en las comunidades sobre todo en aquellas más antiguas, en las cuales el santo patrono o la santa patrona convoca a la gente de la comunidad, a los/as emigrados/as y a las comunidades vecinas. Es el momento del encuentro entre los que fueron vecinos, 
compañeros/as de escuelas, de colegios, de futbol. La añoranza por el valle se disipa en las celebraciones de las fiestas patronales.

La reciprocidad como se ha visto no desaparece en las comunidades nuevas, al contrario, de acuerdo a lo que se pudo observar la misma sigue siendo habitual y en alguna etapa de la formación de la colonia se da de manera más intensa. Las necesidades comunes y las carencias de servicios obligan a las familias a organizarse para proveerse de esas necesidades y a reclamarlas mancomunadamente a las autoridades. En las comunidades más antiguas, la reciprocidad se direcciona hacia las familias más pobres, hacia los familiares y los vecinos más cercanos.

¿Cuálserá elfuturodeestas prácticasen las condiciones actuales de desarrollo de las comunidades en las cuales la cultura urbana, moderna, va permeando progresivamente?. La cooperación interfamiliar, comunitaria ¿podrá perdurar en el futuro? dada la fuerte propaganda y la incursión de la cultura que promueve y premia la iniciativa individual frente a la colectiva. La respuesta a esta pregunta tendría relación con la capacidad de rechazo o de convivencia con la nueva forma de vida que propone el capital. Ciertas prácticas o mecanismos de cooperación siguen vigentes, pero por otra parte, se da un proceso de mayor diferenciación social que es resultado de cambios estructurales dentro de las comunidades, que determinan a su vez, cambios en el modo de ser y de actuar de los pobladores.

\section{Referencias Bibliográficas}

Bartra Verges, A. (2010). Campesindios. Aproximaciones a los campesinos de un continente colonizado. La Paz: Instituto para el Desarrollo Rural de Sudamérica.

Carbonell de Masi, R. (1980). Las cooperativas como forma de organización promocional campesina. En Cooperación de Obras (Ed.), Oñondivepá (p. 59-75). Asunción: Cooperación de Obras.

Domínguez, R. (1977). El valle y la loma.
Asunción: El Lector.

Galeano, L. (1984). Ensayo sobre cultura campesina. Asunción: CPES.

Grünberg, G. (1975). Dos modelos de economía rural en el Paraguay: Pai Tavyterä y Koygua. In: Estudios Paraguayos. Asunción: UCA.

Lehner, B., Pilz, D. \& Riquelme, Q. (2004). Redes de reciprocidad en economías campesinas tradicionales. Asunción: Helvetas-CDE.

Mauss, M. (1971). Ensayo sobre el don. Razón forma del cambio en las sociedades primitivas 1923. Madrid: Editorial Tecnos. www.econ.uba.ar/ www/institutos/economia/Ceplad/ HPE.../Mauss\%2ocastellano.pdf

Meliá, B. \& Temple, D. (2004). El don, la venganza y otras formas de economía guaraní. Asunción: CEPAG.

Meliá, B. (21994). Apuntes de clase. Postgrado en Desarrollo 1994-95. Asunción: CERI-Rectorado-UNA.

Pastoral Social Nacional PSN. (1984). Tierra y Sociedad. Problemática de la tierra urbana, rural e indígena en el Paraguay. Asunción: Pastoral Social Nacional. Cuadernos de Pastoral Social $\mathrm{N}^{\circ} 4$.

Riquelme, Q. \& Vera, E. (2016). Actualización de aspectos legales vinculados a la Agricultura Familiar Campesina (AFC) en Paraguay. Asunción: Decidamos, CDE.

Riquelme, Q. (2014). El derecho a la tierra desde la concepción de los movimientos campesinos. In: P. Dobrée (Ed.), La tierra en el Paraguay: de la desigualdad al ejercicio de derechos (p. 47-62). Asunción: DCP.

Temple, D. (1989). Estructura comunitaria y reciprocidad, del quid-pro-cuo histórico al economicidio. La Paz. Bolivia: Hisbol.

Temple, D. (2003). El don es lo contrario del intercambio. http:// dominique.temple.free.fr/reciprocite. php? page $=$ reciprocidad_ $2 \&$ id article $=442$

Van Dam, C. (2007). Tierra, territorio y 
derechos de los pueblos: indígenas, campesinos y pequeños productores de Salta. Buenos Aires: Secretaría de Agricultura, Ganadería, Pesca y Alimentos.

\author{
Wolf, E. (1977). Una tipología del \\ campesinado latinoamericano. Buenos \\ Aires: Nueva Visión.
}

\section{Sobre los Autores}

Quintín Riquelme

Licenciado en Sociología por la Universidad Católica de Asunción. Docente en las carreras de Trabajo Social y Sociología en la Facultad de Ciencias Sociales (FACSO-UNA). Docente Investigador de la FACSO. Investigador del Centro de Documentación y Estudios (CDE) y actual coordinador del Área Sociogremial de la institución.

Elsy Vera

Licenciada en Ciencias de la Comunicación por la Universidad Nacional de Pilar. Asistente investigadora del Centro de Documentación y Estudios (CDE) de 2001 a 2019. Docente del Instituto de Trabajo Social (ITS-UNA) en el 2015. 